\title{
Entanglement in a Quantum Annealing Processor
}

T. Lanting, ${ }^{1, *}$ A. J. Przybysz, ${ }^{1}$ A. Yu. Smirnov, ${ }^{1}$ F. M. Spedalieri, ${ }^{2,3}$ M. H. Amin,${ }^{1,4}$ A. J. Berkley, ${ }^{1}$ R. Harris, ${ }^{1}$ F. Altomare, ${ }^{1}$ S. Boixo, ${ }^{2,5}$ P. Bunyk, ${ }^{1}$ N. Dickson, ${ }^{1, \dagger}$ C. Enderud, ${ }^{1}$ J. P. Hilton, ${ }^{1}$ E. Hoskinson, ${ }^{1}$ M. W. Johnson, ${ }^{1}$ E. Ladizinsky, ${ }^{1}$ N. Ladizinsky, ${ }^{1}$ R. Neufeld, ${ }^{1}$ T. Oh, ${ }^{1}$ I. Perminov, ${ }^{1}$ C. Rich, ${ }_{1}^{1}$ M. C. Thom, ${ }^{1}$ E. Tolkacheva, ${ }^{1}$ S. Uchaikin, ${ }^{1,6}$ A. B. Wilson, ${ }^{1}$ and G. Rose ${ }^{1}$

${ }^{1}$ D-Wave Systems Inc., 3033 Beta Avenue, Burnaby, British Columbia, Canada V5G $4 M 9$

${ }^{2}$ Information Sciences Institute, University of Southern California, Los Angeles, California 90089, USA

${ }^{3}$ Center for Quantum Information Science and Technology, University of Southern California, Los Angeles, California 90089, USA

${ }^{4}$ Department of Physics, Simon Fraser University, Burnaby, British Columbia, Canada V5A 1S6

${ }^{5}$ Google, 340 Main Street, Venice, California 90291, USA

${ }^{6}$ National Research Tomsk Polytechnic University, 30 Lenin Avenue, Tomsk, 634050, Russia

(Received 13 December 2013; published 29 May 2014)

\begin{abstract}
Entanglement lies at the core of quantum algorithms designed to solve problems that are intractable by classical approaches. One such algorithm, quantum annealing (QA), provides a promising path to a practical quantum processor. We have built a series of architecturally scalable QA processors consisting of networks of manufactured interacting spins (qubits). Here, we use qubit tunneling spectroscopy to measure the energy eigenspectrum of two- and eight-qubit systems within one such processor, demonstrating quantum coherence in these systems. We present experimental evidence that, during a critical portion of QA, the qubits become entangled and entanglement persists even as these systems reach equilibrium with a thermal environment. Our results provide an encouraging sign that QA is a viable technology for largescale quantum computing.
\end{abstract}

DOI: $10.1103 /$ PhysRevX.4.021041

\section{INTRODUCTION}

The past decade has been exciting for the field of quantum computation. A wide range of physical implementations of architectures that promise to harness quantum mechanics to perform computation have been studied [1-3]. Scaling these architectures to build practical processors with many millions to billions of qubits will be challenging [4,5]. A simpler architecture, designed to implement a single quantum algorithm such as quantum annealing (QA), provides a more practical approach in the near term [6,7]. However, one of the main features that makes such an architecture scalable, namely, a limited number of low-bandwidth external control lines [8], prohibits many typical characterization measurements used in studying prototype universal quantum computers [9-14]. These constraints make it challenging to experimentally determine whether a scalable QA architecture, one that is

\footnotetext{
*tanting@dwavesys.com

${ }^{\dagger}$ Present address: Side Effects Software, 1401-123 Front Street West, Toronto, Ontario, Canada.

Published by the American Physical Society under the terms of the Creative Commons Attribution 3.0 License. Further distribution of this work must maintain attribution to the author(s) and the published article's title, journal citation, and DOI.
}

Subject Areas: Quantum Physics,

Quantum Information, Superconductivity

inevitably coupled to a thermal environment, is capable of generating entangled states [15-18]. A demonstration of entanglement is considered to be a critical milestone for any approach to building a quantum computing technology. Herein, we demonstrate an experimental method to detect entanglement in subsections of a quantum annealing processor to address this fundamental question.

\section{QUANTUM ANNEALING}

QA is designed to find the low-energy configurations of systems of interacting spins. A wide variety of optimization problems naturally map onto this physical system [19-22]. A QA algorithm is described by a time-dependent Hamiltonian for a set of $N$ spins, $i=1, \ldots, N$,

$$
\mathcal{H}_{S}(s)=\mathcal{E}(s) \mathcal{H}_{P}-\frac{1}{2} \sum_{i} \Delta(s) \sigma_{i}^{x}
$$

where the dimensionless $\mathcal{H}_{P}$ is

$$
\mathcal{H}_{P}=-\sum_{i} h_{i} \sigma_{i}^{z}+\sum_{i<j} J_{i j} \sigma_{i}^{z} \sigma_{j}^{z}
$$

and $\sigma_{i}^{x, z}$ are Pauli matrices for the $i$ th spin. The energy scales $\Delta$ and $\mathcal{E}$ are the transverse and longitudinal energies 
of the spins, respectively, and the biases $h_{i}$ and couplings $J_{i j}$ encode a particular optimization problem. The timedependent variation of $\Delta$ and $\mathcal{E}$ is parametrized by $s \equiv t / t_{f}$ with time $t \in\left[0, t_{f}\right]$ and total run (anneal) time $t_{f}$. QA is performed by first setting $\Delta \gg \mathcal{E}$, which results in a ground state into which the spins can be easily initialized [6]. Then $\Delta$ is reduced and $\mathcal{E}$ is increased until $\mathcal{E} \gg \Delta$. At this point, the system Hamiltonian is dominated by $\mathcal{H}_{P}$, which represents the encoded optimization problem. At the end of the evolution, a ground state of $\mathcal{H}_{P}$ represents the lowest energy configuration for the problem Hamiltonian and thus a solution to the optimization problem.

\section{QUANTUM ANNEALING PROCESSOR}

We have built a processor that implements $\mathcal{H}_{S}$ using superconducting flux qubits as effective spins $[6,7,23,24]$. Figure 1(a) shows a photograph of the processor. Figure 1(c) shows the circuit schematic of a pair of flux qubits with the magnetic flux controls $\Phi_{q i}^{x}$ and $\Phi_{\text {ccjj }}^{x}$. The annealing parameter $s$ is controlled with the global bias $\Phi_{\text {ccjj }}^{x}(t)$ (see Appendix A for the mapping between $s$ and $\Phi_{\text {ccij }}^{x}$ and a description of how $\Phi_{q i}^{x}$ is provided for each qubit). The strength and sign of the inductive coupling between pairs of qubits is controlled with magnetic flux $\Phi_{\mathrm{co}, i j}^{x}$ that is provided by an individual on-chip digital-to-analog converter for each coupler [8]. The parameters $h_{i}$ and $J_{i j}$ are thus in situ tunable, thereby allowing the encoding of a vast number of problems. The time-dependent energy scales $\Delta(s)$ and $\mathcal{E}(s)$ are calculated from measured qubit parameters and plotted in Fig. 1(d). We calibrate and correct the individual flux qubit parameters in our processor to ensure that every qubit has a close-to-identical $\Delta$ and $\mathcal{E}$ (the energy gap $\Delta$ is balanced to better than $8 \%$ between qubits and $\mathcal{E}$ to better than 5\%). See Appendix A for measurements of these energy scales. The interqubit couplers are calibrated as described in Ref. [25]. The processor we study here is mounted on the mixing chamber of a dilution refrigerator held at temperature $T=12.5 \mathrm{mK}$.

\section{FERROMAGNETICALLY COUPLED INSTANCES}

The experiments reported herein focus on one of the eight-qubit unit cells of the larger QA processor as indicated in Fig. 1(a). The unit cell is isolated by setting all couplings outside of that subsection to $J_{i j}=0$ for all experiments. We then pose specific $\mathcal{H}_{P}$ instances with strong ferromagnetic (FM) coupling $J_{i j}=-2.5$ and $h_{i}=0$ to that unit cell, as illustrated in Figs. 1(e) and 1(f). These configurations produce coupled two- and eight-qubit systems, respectively. The Hamiltonian (1) describes the behavior of these systems during QA.

Typical observations of entanglement in the quantum computing literature involve applying interactions between (a)
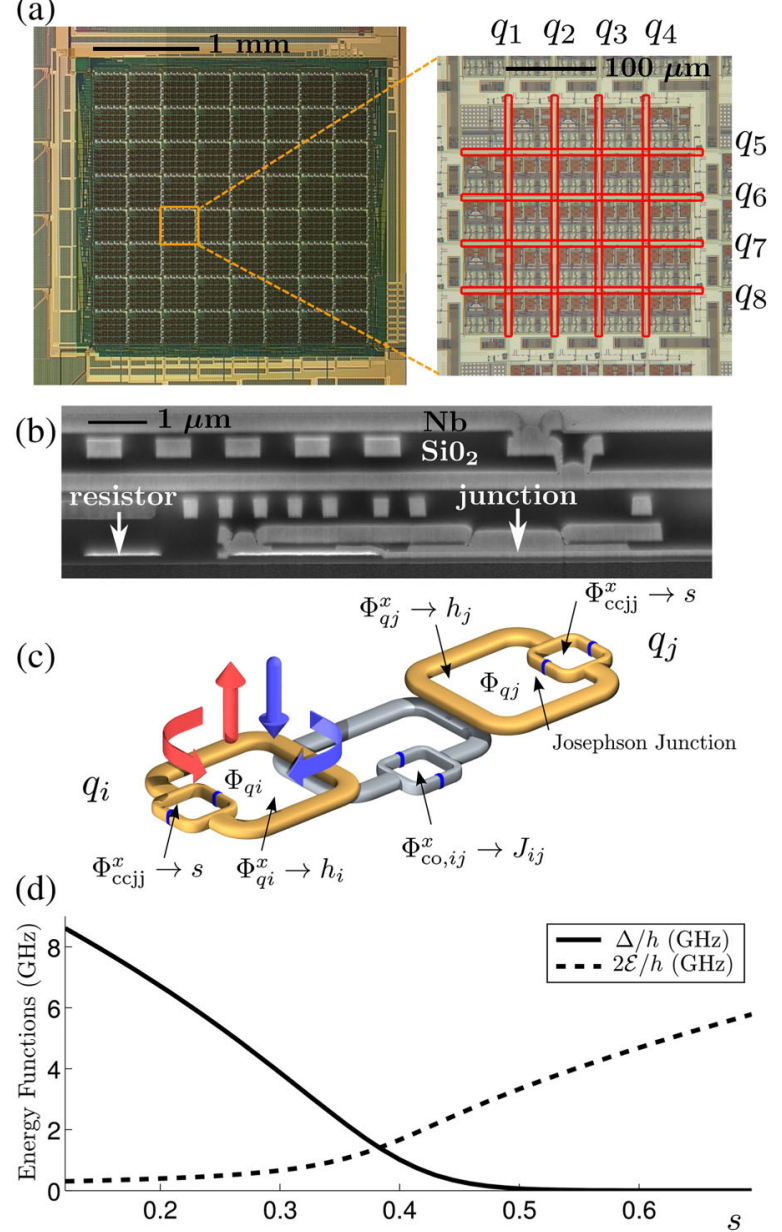

(e)

(f)
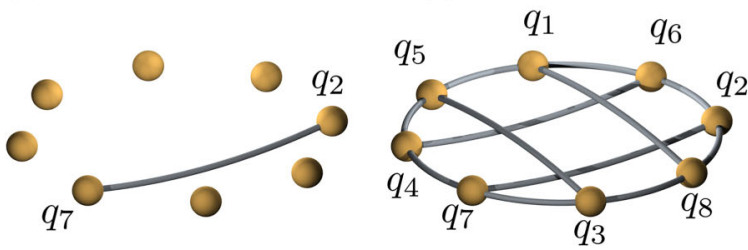

FIG. 1. (a) Photograph of the QA processor used in this study. We report measurements performed on the eight-qubit unit cell indicated. The bodies of the qubits are extended loops of $\mathrm{Nb}$ wiring (highlighted with red rectangles). Interqubit couplers are located at the intersections of the qubit bodies. (b) Electron micrograph showing the cross section of a typical portion of the processor circuitry (described in more detail in Appendix A). (c) Schematic diagram of a pair of coupled superconducting flux qubits with external control biases $\Phi_{q i}^{x}$ and $\Phi_{\text {ccjj }}^{x}$ and with flux through the body of the $i$ th qubit denoted as $\Phi_{q i}$. An inductive coupling between the qubits is tuned with the bias $\Phi_{\mathrm{co}, i j}^{x}$. (d) Energy scales $\Delta(s)$ and $\mathcal{E}(s)$ in Hamiltonian (1) calculated from a rf SQUID model based on the median of independently measured device parameters for these eight qubits. See Appendix A for more details. (e),(f) The two- and eight-qubit systems studied were programmed to have the topologies shown. Qubits are represented as gold spheres, and interqubit couplers, set to $J=-2.5$, are represented as silver lines. 
qubits, removing these interactions, and then performing measurements. Such an approach is well suited to gatemodel architectures (see, e.g., Ref. [11]). During QA, however, the interaction between qubits is determined by the particular instance of $\mathcal{H}_{P}$, in this case, a strongly ferromagnetic instance, and cannot be removed. In this way, systems of qubits undergoing QA have much more in common with condensed-matter systems, such as quantum magnets, for which interactions cannot be turned off. Indeed, a growing body of recent theoretical and experimental work suggests that entanglement plays a central role in many of the macroscopic properties of condensedmatter systems [26-32]. Here, we introduce other approaches to quantifying entanglement that are suited to QA processors. We establish experimentally that the two- and eight-qubit systems, comprising macroscopic

(a)

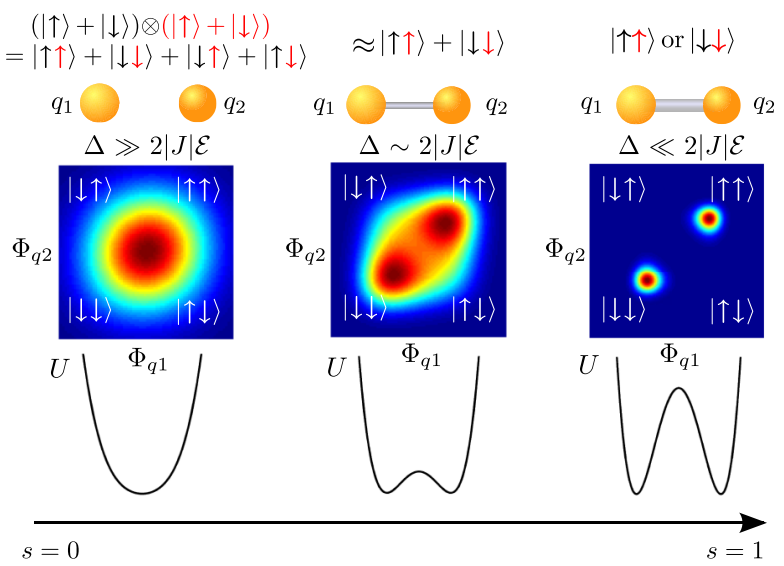

superconducting flux qubits coupled to a thermal bath at $12.5 \mathrm{mK}$, become entangled during the QA algorithm.

To illustrate the evolution of the ground state of these instances during QA, a sequence of wave functions for the ground state of the two-qubit system is shown in Fig. 2. A similar sequence could be envisioned for the eight-qubit system. We consider these systems subject to zero biases, $h_{i}=0$. For small $s, \Delta \gg 2\left|J_{i j}\right| \mathcal{E}$, and the ground state of the system can be expressed as a product of the ground states of the individual qubits: $\otimes_{i=1}^{N}(1 / \sqrt{2})\left(|\uparrow\rangle_{i}+|\downarrow\rangle_{i}\right)$, where $N=2,8$ [see Fig. 2(a)]. For intermediate $s$, $\Delta \lesssim 2\left|J_{i j}\right| \mathcal{E}$, and the ground and first excited states of the processor are approximately the delocalized superpositions $| \pm\rangle \equiv(|\uparrow \ldots \uparrow\rangle \pm|\downarrow \ldots \downarrow\rangle) / \sqrt{2}$ [Fig. 2(b)]. The state $|+\rangle$ is the maximally entangled Bell [or GreenbergerHorne-Zeilinger (GHZ), for eight qubits] state [17]. As $s \rightarrow 1$, the energy gap $g$ between the ground and first excited states approaches $g \equiv\left(E_{2}-E_{1}\right) \propto \Delta(s)^{N} /$ $\left[2\left|J_{i j}\right| \mathcal{E}(s)\right]^{N-1}$ and vanishes as $\Delta(s) \rightarrow 0$ [Fig. 2(c)]. At some point late in the evolution, $g$ becomes less than $k_{B} T$, where $T$ characterizes the temperature of the thermal environment to which the qubits are coupled. At this point, we expect the system to evolve into a mixed state of $|+\rangle$ and $|-\rangle$, and the entanglement will vanish with $g$ for sufficiently long thermalization times. At the end of QA, $s=1, \Delta \sim 0$, and Hamiltonian (1) predicts two degenerate and localized ground states, namely, the FM-ordered states $|\uparrow \ldots \uparrow\rangle$ and $|\downarrow \ldots \downarrow\rangle$.

\section{MEASUREMENTS}

In order to experimentally verify the change in spectral gap in the two- and eight-qubit systems during QA, we use qubit tunneling spectroscopy (QTS), as described in more detail in Ref. [33] and Appendix B. QTS allows us to measure the eigenspectrum and level occupation of a system during QA by coupling an additional probe qubit to the system. We perform QTS on the two- and eight-qubit systems shown in Figs. 1(e) and 1(f). Figures 3(a) and 3(b) show the measured energy eigenspectrum for the two- and eight-qubit systems, respectively, as a function of $s$. The measurements are initial tunneling rates of the probe qubit, normalized by the maximum observed tunneling rate. Peaks in the measured tunneling rate map the energy eigenstates of the system under study [33]. As the system evolves (increasing $s$ ), $\Delta(s)$ in Hamiltonian (1) decreases and the gap between ground and first excited states closes. The spectroscopy data in Fig. 3(a) reveal two higher-energy eigenstates. We observe a similar group of higher-energy excited states for the eight-qubit system in Fig. 3(b). Note that $g$ closes earlier in the QA algorithm for the eight-qubit system as compared to the two-qubit system. In all of the panels of Fig. 3, solid curves indicate the theoretical energy levels predicted by Hamiltonian (1) using the measured 
(a)

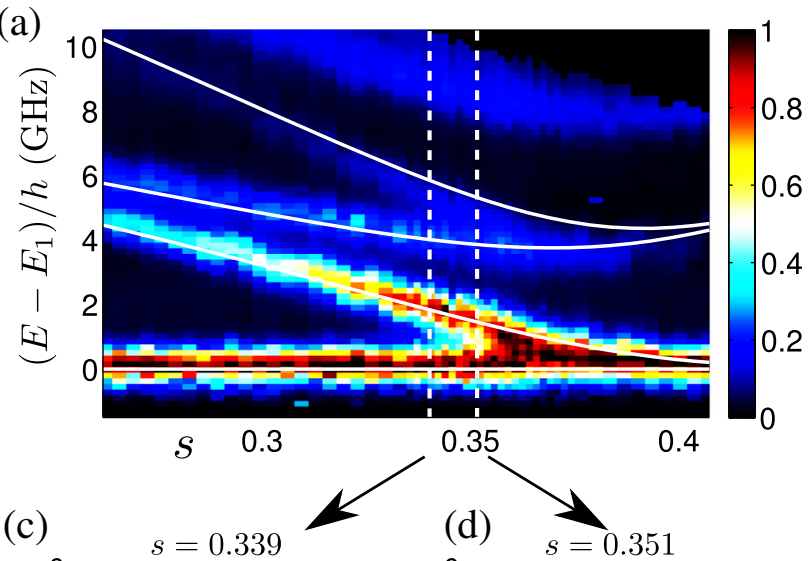

(c) $s=0.339$

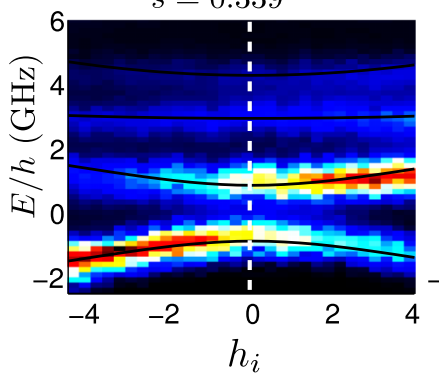

(d) $\quad s=0.351$

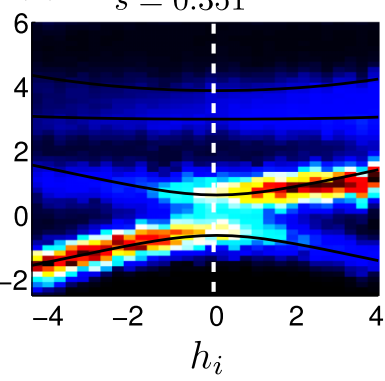

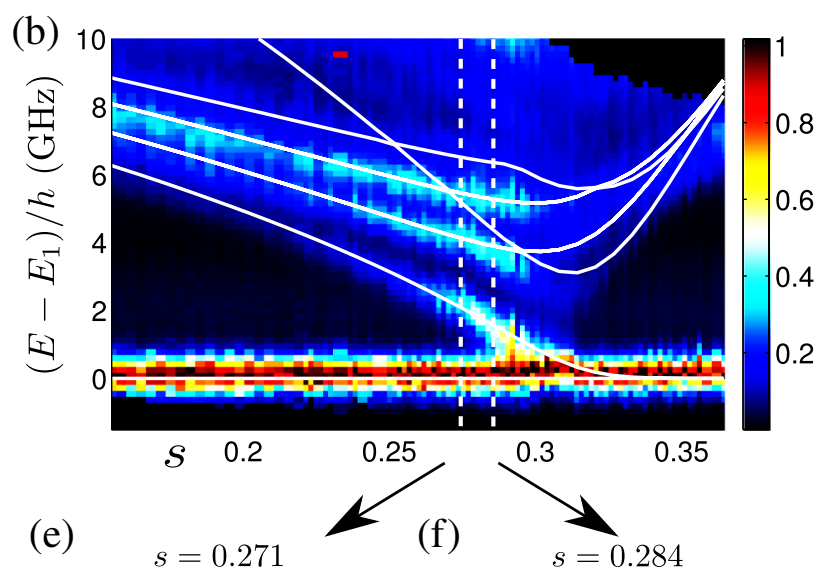

(e)

$s=0.271$
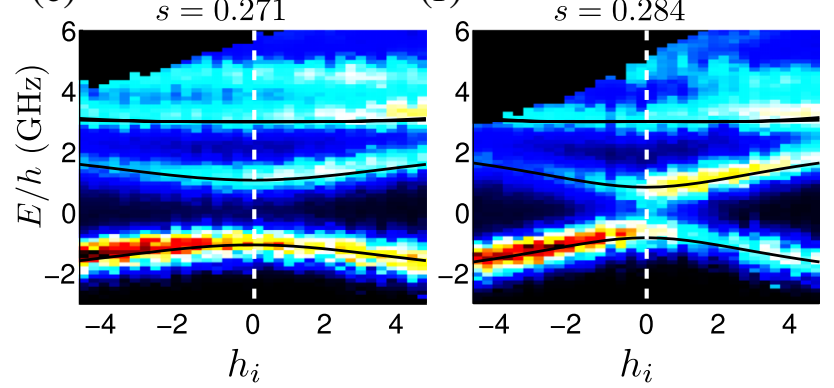

FIG. 3. Spectroscopic data for two- and eight-qubit systems plotted in false color (color indicates normalized qubit tunnel spectroscopy rates). A nonzero measurement (false color) indicates the presence of an eigenstate of the probed system at a given energy (ordinate) and $s$ (abscissa). Panel (a) shows the measured eigenspectrum for the two-qubit system as a function of $s$. Panel (b) shows a similar set of measurements for the eight-qubit system. The ground-state energy $E_{1}$ has been subtracted from the data to aid in visualization. The solid curves indicate the theoretical expectations for the energy eigenvalues using independently calibrated qubit parameters and Hamiltonian (1). We emphasize that the solid curves are not a fit, but rather a prediction based on Hamiltonian (1) and measurements of $\Delta$ and $\mathcal{E}$. The slight differences between the high-energy spectrum prediction and measurements are due to the additional states in the rf SQUID flux qubits. A full rf SQUID model that is in agreement with the measured high-energy spectrum is explored in the Supplemental Material [34]. Panels (c) and (d) show measured eigenspectra of the two-qubit system versus $h_{1}=h_{2} \equiv h_{i}$ for two values of annealing parameter $s, s=0.339$ and $s=0.351$ from left to right, respectively. Note the avoided crossing at $h_{i}=0$. Panels (e) and (f) show analogous measured eigenspectra for the eight-qubit system (with $h_{1}=\cdots=h_{8} \equiv h_{i}$ ). Because the eight-qubit gap closes earlier in QA for this system, we show measurements for smaller $s, s=0.271$ and $s=0.284$ from left to right, respectively.

$\Delta(s)$ and $\mathcal{E}(s)$. The agreement between the experimentally obtained spectrum and the theoretical spectrum is good.

The data presented in Figs. 3(a) and 3(b) indicate that the spectral gap between ground and first excited state decreases monotonically with $s$ when all $h_{i}=0$. Under these bias conditions, these systems possess $Z_{2}$ symmetry between the states $|\uparrow \ldots \uparrow\rangle$ and $|\downarrow \ldots \downarrow\rangle$. The degeneracy between these states is lifted by finite $\Delta(s)$. To explicitly demonstrate that the spectral gap at $h_{i}=0$ is due to the avoided crossing of $|\uparrow \ldots \uparrow\rangle$ and $|\downarrow \ldots \downarrow\rangle$, we perform QTS at fixed $s$ as a function of a "diagnostic" bias $h_{i} \neq 0$ that was uniformly applied to all qubits, thus sweeping the systems through degeneracy at $h_{i}=0$. As a result, either the state $|\uparrow \ldots \uparrow\rangle$ or $|\downarrow \ldots \downarrow\rangle$ becomes energetically favored, depending upon the sign of $h_{i}$. Hamiltonian (1) predicts an avoided crossing, as a function of $h_{i}$, between the ground and first excited states at degeneracy, where $h_{i}=0$, with a minimum energy gap $g$. The presence of such an avoided crossing is a signature of ground-state entanglement [14,35]. For large gaps, $g>k_{B} T$, there is persistent entanglement at equilibrium (see Refs. [18,26,28,29,31]).
We experimentally verify the existence of avoided crossings at multiple values of $s$ in both the two- and eight-qubit systems by using QTS across a range of biases $h_{i} \in\{-4,4\}$. In Fig. 3(c), we show the measured spectrum of the two-qubit system at $s=0.339$ up to an energy of $6 \mathrm{GHz}$ for a range of bias $h_{i}$. The ground states at the far left and far right of the spectrum are the localized states $|\downarrow \downarrow\rangle$ and $|\uparrow \uparrow\rangle$, respectively. At $h_{i}=0$, we observe an avoided crossing between these two states. We measure an energy gap $g$ at zero bias, $h_{i}=0$, between the ground state and the first excited state, $g / h=1.75 \pm 0.08 \mathrm{GHz}$, by fitting a Gaussian profile to the tunneling rate data at these two lowest-energy levels and subtracting the centroids. Here, $h$ (without any subscript) is the Planck constant. Figure 3(d) shows the two-qubit spectrum later in the QA algorithm, at $s=0.351$. The energy gap has decreased to $g / h=$ $1.21 \pm 0.06 \mathrm{GHz}$. Note that the error estimates for the energy gaps are derived from the uncertainty in extracting the centroids from the rate data. We discuss the actual source of the underlying Gaussian widths (the observed level broadening) below. For both the two- and eight-qubit 
(a)

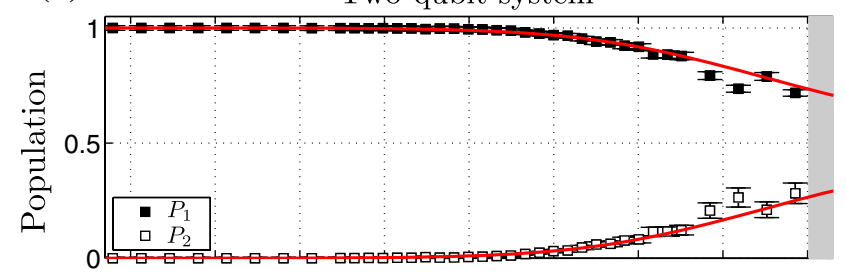

(c)

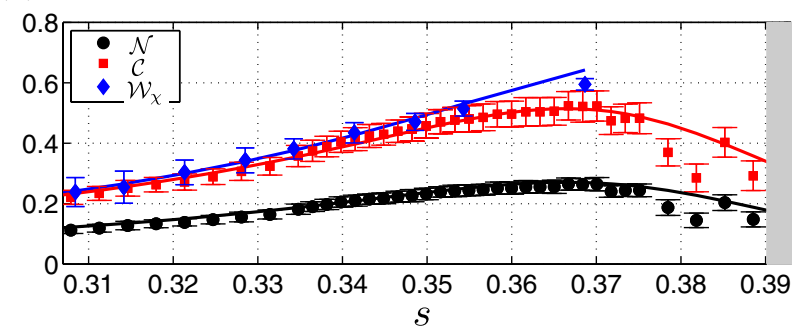

(b) Eight-qubit system

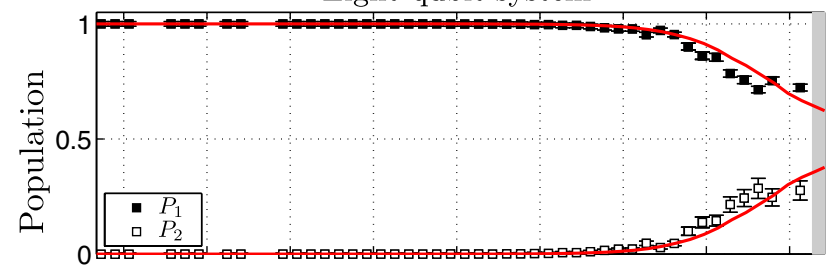

(d)

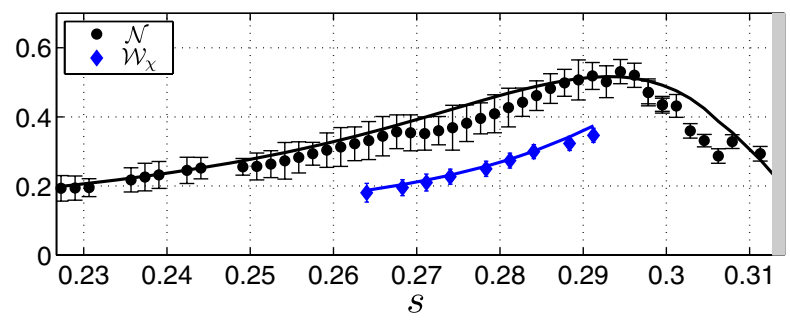

FIG. 4. (a),(b) Measurements of the occupation fraction, or population, of the ground state $\left(P_{1}\right)$ and first excited state $\left(P_{2}\right)$ of the twoqubit and eight-qubit system, respectively, versus $s$. Early in the annealing trajectory, $g \gg k_{B} T$, and the system is in the ground state with $P_{1} \lesssim 1$. The solid curves show the equilibrium Boltzmann predictions for $T=12.5 \mathrm{mK}$. (c) Concurrence $\mathcal{C}$, negativity $\mathcal{N}$, and witness $\mathcal{W}_{\chi}$ versus $s$ for the two-qubit system. Early in QA, the qubits are weakly interacting, thus resulting in limited entanglement. Entanglement peaks near $s=0.37$. For larger $s$, the gap between the ground and first excited state shrinks and thermal occupation of the first excited state rises, thus extinguishing entanglement. Solid curves indicate the expected theoretical values of each witness or measure using Hamiltonian (1) and Boltzmann statistics. (d) Negativity $\mathcal{N}$ and witness $\mathcal{W}_{\chi}$ versus $s$ for the eight-qubit system. For all $s$ shown, the nonzero negativity $\mathcal{N}$ and nonzero witness $\mathcal{W}_{\chi}$ report entanglement. For $s>0.39$ and $s>0.312$ for the two-qubit and eight-qubit systems, respectively, the shaded gray region denotes the regime in which the ground and first excited states cannot be resolved via our spectroscopic method. Solid curves indicate the expected theoretical values of each witness or measure using Hamiltonian (1) and Boltzmann statistics.

systems, we confirm that the expectation values of $\sigma_{z}$ for all devices change sign as the system moves through the avoided crossing (see Figs. 1-3 of the Supplemental Material [34] and Ref. [35]).

Figures 3(e) and 3(f) show similar measurements of the spectrum of eight coupled qubits at $s=0.271$ and $s=$ 0.284 for a range of biases $h_{i}$. Again, we observe an avoided crossing at $h_{i}=0$. The measured energy gaps at $s=0.271$ and 0.284 are $g / h=2.2 \pm 0.08 \mathrm{GHz}$ and $g / h=1.66 \pm 0.06 \mathrm{GHz}$, respectively. Although the eightqubit gaps in Figs. 3(e) and 3(f) are close to the two-qubit gaps in Figs. 3(c) and 3(d), they are measured at quite different values of the annealing parameter $s$. As expected, the eight-qubit gap is closing earlier in the QA algorithm as compared to the two-qubit gap. The solid curves in Figs. 3(c)-3(f) indicate the theoretical energy levels predicted by Hamiltonian (1) and measurements of $\Delta(s)$ and $\mathcal{E}(s)$. Again, the agreement between the experimentally obtained spectra and the theoretical spectra is good.

For the early and intermediate parts of QA, the energy gap $g$ is larger than temperature, $g \gg k_{B} T$, for both the two- and eight-qubit systems. We expect that if we hold the systems at these $s$, then the only eigenstate with significant occupation will be the ground state. We confirm this by using QTS in the limit of long tunneling times to probe the occupation fractions. Details are provided in Appendix C. Figures 4(a) and 4(b) show the measured occupation fractions of the ground and first excited states as a function of $s$ for both the two- and eight-qubit systems. The solid curves show the equilibrium Boltzmann predictions for $T=12.5 \mathrm{mK}$ and are in good agreement with the data.

The width of the measured spectral lines is dominated by the noise of the probe device used to perform QTS [33]. The probe device is operated in a regime in which it is strongly coupled to its environment, whereas the system qubits we study are in the weak coupling regime. The measured line widths, which do not increase with system size, therefore, do not represent the intrinsic width of the two- and eight-qubit energy eigenstates. During the intermediate part of QA, the ground and first excited states are clearly resolved. The ground state is protected by the multiqubit energy gap $g \gg k_{B} T$, and these systems are coherent. At the end of the annealing trajectory, the gap between the ground state and first excited state shrinks below the probe qubit line width of $0.4 \mathrm{GHz}$. An analysis of spectroscopic data, presented in the Supplemental Material [34], shows that the intrinsic energy levels remain distinct until later in QA. The interactions between the two- and eight-qubit systems and their respective environments represent small perturbations to Hamiltonian (1), even in 
the regime in which entanglement is beginning to fall due to thermal mixing.

\section{ENTANGLEMENT DETECTION}

The tunneling spectroscopy data show that, midway through QA, both the two- and eight-qubit systems have avoided crossings with the expected gap $g \gg k_{B} T$ and have ground-state occupation $P_{1} \simeq 1$. While observation of an avoided crossing is evidence for the presence of an entangled ground state [35], we can make this observation more quantitative with entanglement measures and witnesses.

For a large part of the QA algorithm, the two- and eightqubit systems are in their ground states with high occupation fraction. We, therefore, begin the analysis with a susceptibility-based witness $\mathcal{W}_{\chi}$, which detects groundstate entanglement. This witness does not require explicit knowledge of Hamiltonian (1), but requires a nondegenerate ground state, confirmed with the avoided crossings shown in Fig. 3, and high occupation fraction of the ground state, confirmed early in QA by the measurements of $P_{1} \simeq 1$ shown in Fig. 4. We then perform measurements of all available linear cross susceptibilities

$$
\chi_{i j} \equiv d\left\langle\sigma_{i}^{z}\right\rangle / d \tilde{h}_{j},
$$

where $\left\langle\sigma_{i}^{z}\right\rangle$ is the expectation value of $\sigma_{i}^{z}$ for the $i$ th qubit and $\tilde{h}_{j}=\mathcal{E} h_{j}$ is a bias applied to the $j$ th qubit. The measurements are performed at the degeneracy point (in the middle of the avoided crossings), where the classical contribution to the cross susceptiblity is zero.

From these measurements, we calculate $\mathcal{W}_{\chi}$ as defined in Ref. [35] (see Appendix D for more details). A nonzero value of this witness detects ground-state entanglement, and global entanglement in the case of the eight-qubit system (meaning every possible bipartition of the eightqubit system is entangled). Figures 4(c) and 4(d) show $\mathcal{W}_{\chi}$ for the two- and eight-qubit systems. Note that for two qubits at degeneracy, $\mathcal{W}_{\chi}$ coincides with ground-state concurrence. These results indicate that the two- and eight-qubit systems are entangled midway through QA. Note also that a susceptibility-based witness has a close analogy to susceptibility-based measurements of nanomagnetic systems that also report strong nonclassical correlations $[29,31]$.

The occupation fraction measurements shown in Fig. 4 indicate that midway through QA, the first excited state of these systems is occupied as the energy gap $g$ begins to approach $k_{B} T$. The systems are no longer in the ground state but, rather, in a mixed state. To detect the presence of mixed-state entanglement, we need knowledge about the density matrix of these systems. Occupation fraction measurements provide measurements of the diagonal elements of the density matrix in the energy basis. We assume that the density matrix has no off-diagonal elements in the energy basis (they decay on time scales of several nanoseconds). We relax this assumption below. Populations $P_{1}$ and $P_{2}$ plotted in Figs. 4(a) and 4(b) indicate that the system occupies these states with almost $100 \%$ probability. This means that the density matrix can be written in the form $\rho=\sum_{i=1}^{2} P_{i}\left|\psi_{i}\right\rangle\left\langle\psi_{i}\right|$, where $\left|\psi_{i}\right\rangle$ represents the $i$ th eigenstate of Hamiltonian (1).

We use the density matrix to calculate standard entanglement measures, Wootters' concurrence $\mathcal{C}[18]$ for the two-qubit system, and negativity $\mathcal{N}[16,36]$ for the two- and eight-qubit system. For the maximally entangled two-qubit Bell state, we note that $\mathcal{C}=1$ and $\mathcal{N}=0.5$. Figure 4(c) shows $\mathcal{C}$ as a function of $s$. Midway through $\mathrm{QA}$, we measure a peak concurrence $\mathcal{C}=0.53 \pm 0.05$, indicating significant entanglement in the two-qubit system. This value of $\mathcal{C}$ corresponds to an entanglement of formation $E_{f}=0.388$ (see Refs. [16,18] for definitions). This is comparable to the level of entanglement, $E_{f}=0.378$, obtained in Ref. [11]. Because concurrence $\mathcal{C}$ is not applicable to more than two qubits, we use negativity $\mathcal{N}$ to detect entanglement in the eight-qubit system. For $N>2, \mathcal{N}_{A, B}$ is defined on a particular bipartition of the system into subsystems $A$ and $B$. We define $\mathcal{N}$ to be the geometric mean of this quantity across all possible bipartitions. A nonzero $\mathcal{N}$ indicates the presence of global entanglement. Figures 4(c) and 4(d) show the negativity calculated with measured $P_{1}$ and $P_{2}$ (and with the measured Hamiltonian parameters $\Delta$ and $\mathcal{E} J_{i j}$ ) as a function of $s$ for the two- and eight-qubit systems. The eight-qubit system has nonzero $\mathcal{N}$ for $s<0.315$, thus indicating the presence of mixed-state global entanglement. Both concurrence $\mathcal{C}$ and negativity $\mathcal{N}$ decrease later in QA, where the first excited state approaches the ground state and becomes thermally occupied. The experimental values of these entanglement measures are in agreement with the theoretical predictions (solid lines in Fig. 4). The error bars in Figs. 4(c) and 4(d) represent uncertainties in the measurements of $P_{1}(s), P_{2}(s), \Delta(s)$, and $\mathcal{E}(s)$.

As stated above, the calculation of $\mathcal{C}$ and $\mathcal{N}$ relies on the assumption that the off-diagonal terms in the density matrix decay on time scales of several nanoseconds. We remove this assumption and demonstrate entanglement through the use of another witness $\mathcal{W}_{A B}$, defined on some bipartition $A-B$ of the eight-qubit system. The witness, described in Appendix D, is designed in such a way that $\operatorname{Tr}\left[\mathcal{W}_{A B} \sigma\right] \geq 0$ for all separable states $\sigma$. When $\operatorname{Tr}\left[\mathcal{W}_{A B} \rho(s)\right]<0$, the state $\rho(s)$ is entangled. Measurements of populations $P_{1}$ and $P_{2}$ provide a set of linear constraints on the density matrix of the system $\rho(s)$. We then obtain an upper bound on $\operatorname{Tr}\left[\mathcal{W}_{A B} \rho(s)\right]$ by searching over all $\rho(s)$ that satisfy these linear constraints. If this upper bound is $<0$, then we have shown entanglement for the bipartition $A-B$ [37]. Figure 5 shows the upper limit of the witness $\operatorname{Tr}\left[\mathcal{W}_{A B} \rho(s)\right]$ for the eight-qubit system. We plot data for the bipartition that gives the median upper limit. The error bars are derived 


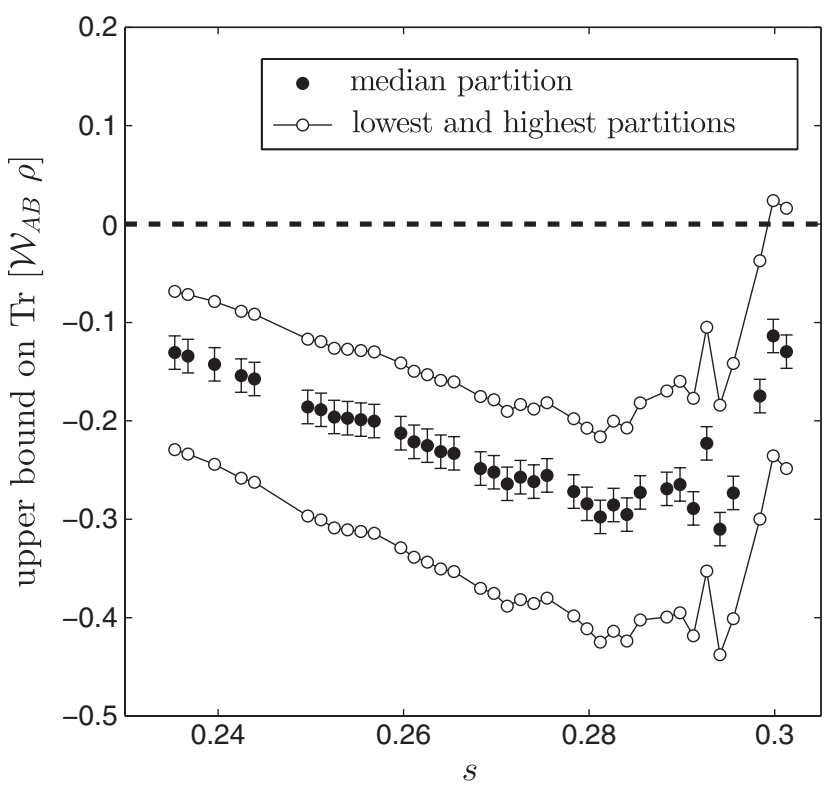

FIG. 5. Upper limit of the quantity $\operatorname{Tr}\left[\mathcal{W}_{A B} \rho\right]$ versus $s$ for several bipartitions $A-B$ of the eight-qubit system. When this quantity is less than 0 , the system is entangled with respect to this bipartition. The solid dots show the upper limit on $\operatorname{Tr}\left[\mathcal{W}_{A B} \rho\right]$ for the median bipartition. The open dots above and below these are derived from the two bipartitions that give the highest and lowest upper limits on $\operatorname{Tr}\left[\mathcal{W}_{A B} \rho\right]$, respectively. For the points at $s>0.3$, the measurements of $P_{1}$ and $P_{2}$ do not constrain $\rho$ enough to certify entanglement.

from a Monte Carlo analysis wherein we used the experimental uncertainties in $\Delta$ and $J$ to estimate the uncertainty in $\operatorname{Tr}\left[\mathcal{W}_{A B} \rho\right]$. We also plot data for the two partitions that give the largest and smallest upper limits. For all values of the annealing parameter $s$, except for the last two points, upper limits from all possible bipartitions of the eight-qubit system are below zero. In this annealing range, the eightqubit system is globally entangled.

\section{CONCLUSIONS}

In summary, we provide experimental evidence for the presence of quantum coherence and entanglement within subsets of qubits inside a quantum annealing processor during its operation. Our conclusion is based on four levels of evidence: (a) the observation of two- and eight- qubit avoided crossings with a multiqubit energy gap $g \gg k_{B} T$; (b) the witness $\mathcal{W} \chi$, calculated with measured cross susceptibilities and coupling energies, which reports ground-state entanglement of the two- and eight-qubit systems [note that these two levels of evidence do not require explicit knowledge of Hamiltonian (1)]; (c) the measurements of energy eigenspectra and equibrium occupation fractions during QA, which allow us to use Hamiltonian (1) to reconstruct the density matrix, with some weak assumptions, and calculate concurrence and negativity (these standard measures of entanglement report nonclassical correlations in the two- and eight-qubit systems); (d) the entanglement witness $\mathcal{W}_{A B}$, which is calculated with the measured Hamiltonian and with constraints provided by the measured populations of the ground and the first excited states (this witness reports global entanglement of the eight-qubit system midway through the QA algorithm).

The observed entanglement is persistent at thermal equilibrium, an encouraging result as any practical hardware designed to run a quantum algorithm will be inevitably coupled to a thermal environment. The experimental techniques we discuss provide measurements of energy levels, and their populations, for arbitrary configurations of Hamiltonian parameters $\Delta, h_{i}, J_{i j}$ during the QA algorithm. The main limitation of the technique is the spectral width of the probe device. Improved designs of this device will allow much larger systems to be studied. Our measurements represent an effective approach for exploring the role of quantum mechanics in QA processors and ultimately to understanding the fundamental power and capability of quantum annealing.

\section{ACKNOWLEDGMENTS}

We thank C. Williams, P. Love, and J. Whittaker for useful discussions. We acknowledge F. Cioata and P. Spear for the design and maintenance of electronics control systems, J. Yao for fabrication support, and D. Bruce, P. deBuen, M. Gullen, M. Hager, G. Lamont, L. Paulson, C. Petroff, and A. Tcaciuc for technical support. F. M. S. was supported by DARPA, under Contract No. FA8750-132-0035.

\section{APPENDIX A: QA PROCESSOR DESCRIPTION}

\section{Chip description}

The experiments discussed in herein were performed on a sample fabricated with a process consisting of a standard $\mathrm{Nb} / \mathrm{AlOx} / \mathrm{Nb}$ trilayer, a TiPt resistor layer, planarized $\mathrm{SiO}_{2}$ dielectric layers, and six $\mathrm{Nb}$ wiring layers. The circuit design rules include a minimum linewidth of $0.25 \mu \mathrm{m}$ and 0.6- $\mu \mathrm{m}$-diameter Josephson junctions. The processor chip is a network of densely connected eight-qubit unit cells that are more sparsely connected to each other (see Fig. 1 for photographs of the processor). We report measurements made on qubits from one of these unit cells. The chip is mounted on the mixing chamber of a dilution refrigerator inside an Al superconducting shield and temperature controlled at $12.5 \mathrm{mK}$.

\section{Qubit parameters}

The processor facilitates quantum annealing of compound-compound Josephson junction rf SQUID flux qubits [38]. The qubits are controlled via the external flux biases $\Phi_{q i}^{x}$ and $\Phi_{\text {ccjj }}^{x}$, which allow us to treat them as effective spins (see Fig. 1). Pairs of qubits interact through tunable 
inductive couplings [25]. The system can be described with the time-dependent QA Hamiltonian,

$$
\mathcal{H}_{S}(s)=\mathcal{E}(s)\left[-\sum_{i}^{N} h_{i} \sigma_{i}^{z}+\sum_{i<j} J_{i j} \sigma_{i}^{z} \sigma_{j}^{z}\right]-\frac{1}{2} \Delta(s) \sum_{i}^{N} \sigma_{i}^{x},
$$

where $\sigma_{i}^{x, z}$ are Pauli matrices for the $i$ th qubit, $i=1, \ldots, N$. The energy scales $\Delta$ and $\mathcal{E}$ are the transverse and longitudinal energies of the spins, respectively, and the unitless biases $h_{i}$ and couplings $J_{i j}$ encode a particular optimization problem. We define $\tilde{h}_{i} \equiv \mathcal{E} h_{i}$ and $\tilde{J}_{i j} \equiv \mathcal{E} J_{i j}$. We map the annealing parameter $s$ for this particular chip to a range of $\Phi_{\mathrm{ccjj}}^{x}$ with the relation

$$
s \equiv\left(\Phi_{\mathrm{ccjj}}^{x}(t)-\Phi_{\mathrm{ccjj}, \text { initial }}^{x}\right) /\left(\Phi_{\mathrm{ccjj}, \text { final }}^{x}-\Phi_{\mathrm{ccjj}, \text { initial }}^{x}\right)=t / t_{f},
$$

where $t_{f}$ is the total anneal time. We implement QA for this processor by ramping the external control $\Phi_{\text {ccjj }}^{x}(t)$ from $\Phi_{\text {ccjj,initial }}^{x}=0.596 \Phi_{0}(s=0)$ at $t=0$ to $\Phi_{\text {ccjj, final }}^{x}=0.666$ $\Phi_{0}(s=1)$ at $t=t_{f}$. The energy scale $\mathcal{E} \equiv M_{\text {eff }}\left|I_{q}^{p}(s)\right|^{2}$ is set by the $s$-dependent persistent current of the qubit $\left|I_{q}^{p}(s)\right|$ and the maximum mutual inductance between qubits $M_{\text {eff }}=1.37 \mathrm{pH}$ [8]. The transverse term in Hamiltonian (A1), $\Delta(s)$, is the energy gap between the ground and first excited state of an isolated rf SQUID at zero bias. $\Delta$ also changes with annealing parameter $s . \Phi_{q i}^{x}(t)$ is provided by a global external magnetic flux bias along with local in situ tunable DAC that tunes the coupling strength of this global bias into individual qubits and thus allows us to specify individual biases $h_{i}$. The coupling energy between the $i$ th and $j$ th qubit is set with a local in situ tunable DAC that controls $\Phi_{\mathrm{co}, i j}^{x}$.

The main quantities associated with a flux qubit, $\Delta$ and $\left|I_{q}^{p}\right|$, primarily depend on macroscopic rf SQUID parameters: junction critical current $I_{c}$, qubit inductance $L_{q}$, and qubit capacitance $C_{q}$. We calibrate all of these parameters on this chip as described in Refs. [6,8]. We calibrate all interqubit coupling elements across their available tuning range from $1.37 \mathrm{pH}$ to $-3.7 \mathrm{pH}$, as described in Ref. [25]. We correct for variations in qubit parameters with on-chip control as described in Ref. [8]. This allows us to match $\left|I_{q}^{p}\right|$ and $\Delta$ across all qubits throughout the annealing trajectory.

TABLE I. Qubit parameters.

\begin{tabular}{lc}
\hline \hline Qubit parameter & Median measured value \\
\hline Critical current, $I_{c}$ & $2.89 \mu \mathrm{A}$ \\
Qubit inductance, $L_{q}$ & $344 \mathrm{pH}$ \\
Qubit capacitance, $C_{q}$ & $110 \mathrm{fF}$ \\
\hline \hline
\end{tabular}
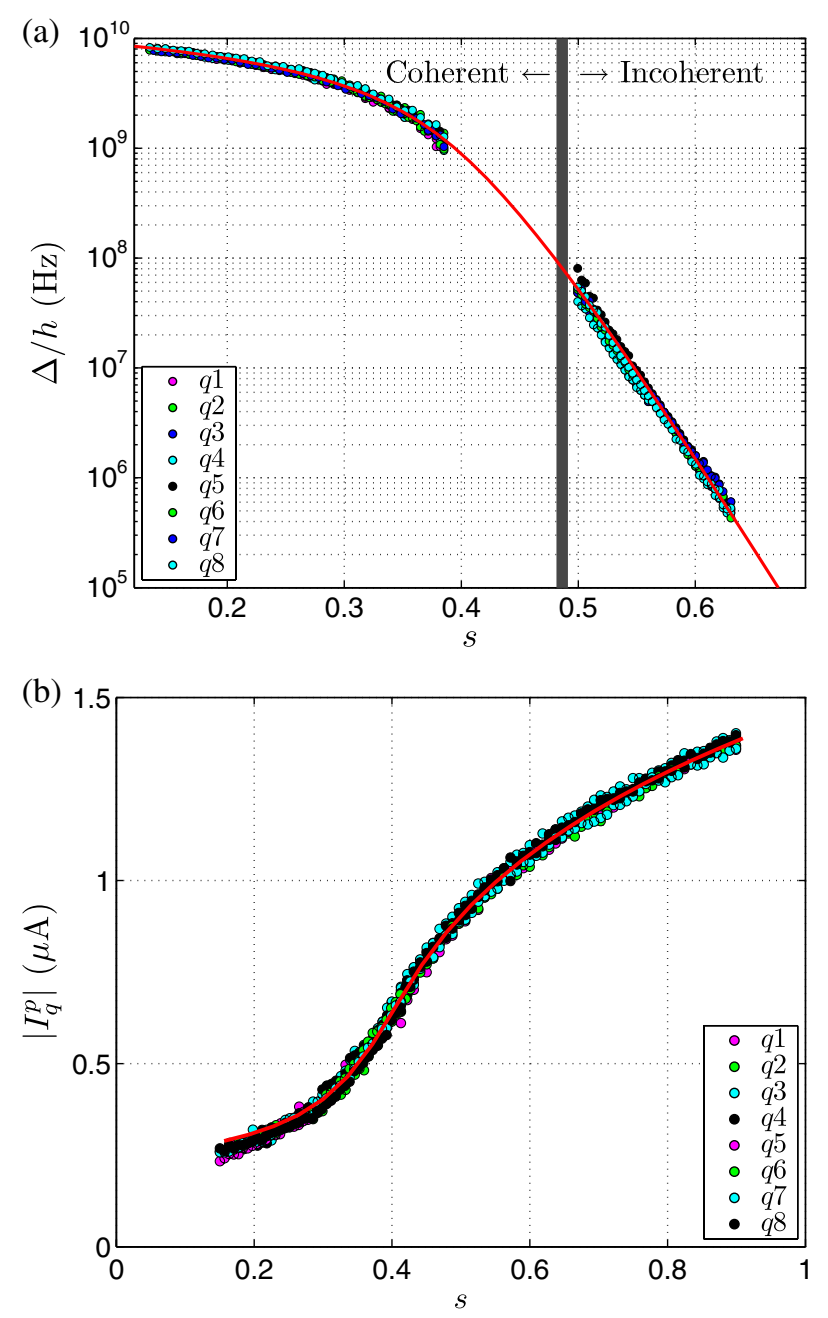

FIG. 6. (a) $\Delta(s)$ versus $s$. We show measurements for all eight qubits studied in this work. We use a single-qubit Landau-Zener experiment to measure $\Delta / h<100 \mathrm{MHz}$ [39]. We use QTS to measure $\Delta / h>1 \mathrm{GHz}$ [33]. The red line shows the theoretical prediction for a rf SQUID model employing the median qubit parameters of the eight devices. The vertical black line separates coherent (left) and incoherent (right) evolution as estimated by analysis of single-qubit spectral line shapes. (b) $\left|I_{q}^{p}\right|(s)$ versus $s$. We show measurements for all eight qubits studied in this work. We use a two-qubit coupled flux measurement with the interqubit coupling element set to $1.37 \mathrm{pH}$ [8]. The red line shows the theoretical prediction for a rf SQUID model employing the median qubit parameters of the eight devices.

Table I shows the median qubit parameters for the devices studied here.

Figure 6 shows measurements of $\Delta$ and $\left|I_{q}^{p}\right|$ versus $s$ for all eight qubits. $\Delta$ is measured with single-qubit LandauZener measurements from $s=0.515$ to $s=0.658$ [39] and with qubit tunneling spectroscopy from $s=0.121$ to $s=$ 0.4 [33]. The resolution limit of qubit tunneling spectroscopy and the bandwidth of our external control lines during the Landau-Zener measurements prevent us from characterizing $\Delta$ between $s=0.4$ and $s=0.5$, respectively. $\left|I_{q}^{p}\right|$ is 
measured by coupling a second probe qubit to the qubit $q_{i}$ with a coupling of $M_{\text {eff }}=1.37 \mathrm{pH}$ and measuring the flux $M_{\text {eff }}\left|I_{q i}^{p}(s)\right|$ as a function of $s .\left|I_{q}^{p}\right|$ is matched between qubits to within $3 \%$ and $\Delta(s)$ is matched between qubits to within $8 \%$ across the annealing region explored in this study.

\section{APPENDIX B: QUBIT TUNNELING SPECTROSCOPY}

QTS allows one to measure the eigenspectrum of an $N$ qubit system governed by Hamiltonian $\mathcal{H}_{S}$. Details on the measurement technique are presented elsewhere [33]. For convenience in comparing with this reference, we define a qubit energy bias $\epsilon_{i} \equiv 2 \tilde{h}_{i}$. Measurements are performed by coupling an additional probe qubit $q_{P}$, with qubit tunneling amplitude $\Delta_{P} \ll \Delta,|\tilde{J}|$, to one of the $N$ qubits of the system under study, for example, $q_{1}$. When we use a coupling strength $\tilde{J}_{P}$ between $q_{P}$ and $q_{1}$ and apply a compensating bias $\epsilon_{1}=2 \tilde{J}_{P}$ to $q_{1}$, the resulting system plus probe Hamiltonian becomes

$$
H_{S+P}=H_{S}-\left[\tilde{J}_{P} \sigma_{1}^{z}-(1 / 2) \epsilon_{P}\right]\left(1-\sigma_{P}^{z}\right) .
$$

For one of the localized states of the probe qubit, $|\uparrow\rangle_{P}$, for which an eigenvalue of $\sigma_{P}^{z}$ is equal to +1 (i.e., the probe qubit in the right well), the contribution of the probe qubit is exactly canceled, leading to $H_{S+P}=H_{S}$, with composite eigenstates $|n, \uparrow\rangle=|n\rangle \otimes|\uparrow\rangle_{P}$ and eigenvalues $E_{n}^{R}=E_{n}$, which are identical to those of the original system without the presence of the probe qubit. Here, $|n\rangle$ is an eigenstate of the Hamiltonian $H_{S}\left(n=1,2, \ldots, 2^{N}\right)$.

For the other localized state of the probe qubit, $|\downarrow\rangle_{P}$, when this qubit is in the left well, the ground state of $H_{S+P}$ is $\left|\psi_{0}^{L}, \downarrow\right\rangle=\left|\psi_{0}^{L}\right\rangle \otimes|\downarrow\rangle_{P}$, with eigenvalue $\tilde{E}_{0}^{L}=E_{0}^{L}+\epsilon_{P}$, where $\left|\psi_{0}^{L}\right\rangle$ is the ground state of $H_{S}-2 \tilde{J}_{P} \sigma_{1}^{z}$ and $E_{0}^{L}$ is its eigenvalue. We choose $\left|\tilde{J}_{P}\right| \gg k_{B} T$ such that the state $\left|\psi_{0}^{L}, \downarrow\right\rangle$ is well separated from the next excited state for ferromagnetically coupled systems, and, thus, system plus probe can be initialized in this state to high fidelity.

Introducing a small transverse term, $-\frac{1}{2} \Delta_{P} \sigma_{P}^{x}$, to Hamiltonian (B1) results in incoherent tunneling from the initial state $\left|\psi_{0}^{L}, \downarrow\right\rangle$ to any of the available $|n, \uparrow\rangle$ states [40]. A bias on the probe qubit $\epsilon_{P}$ changes the energy difference between the probe $|\downarrow\rangle_{P}$ and $|\uparrow\rangle_{P}$ manifolds. We can thus bring $\left|\psi_{0}^{L}, \downarrow\right\rangle$ into resonance with any of $|n, \uparrow\rangle$ states (when $\tilde{E}_{0}^{L}=E_{n}^{R}$ ), allowing resonant tunneling between the two states. The rate of tunneling out of the initially prepared state $\left|\psi_{0}^{L}, \downarrow\right\rangle$ is thus peaked at the locations of $|n, \uparrow\rangle$.

The measurement of the eigenspectrum of an $N$-qubit system thus proceeds as follows. We couple an additional probe qubit to one of the $N$ qubits (say, to $q_{1}$ ) with coupling constant $\tilde{J}_{P}$. We prepare the $(N+1)$-qubit system in the state $\left|\psi_{0}^{L}, \downarrow\right\rangle$ by annealing from $s=0$ to $s=1$ in the presence of large bias $\epsilon_{\mathrm{pol}}<0$ on all the system and probe qubits. We then adjust $s$ for the $N$-qubit system to an intermediate point $s^{*} \in[0,1]$, such that $\Delta \gg k_{B} T / h$, and adjust $s$ for the probe qubit to $s_{P}=0.612$, such that $\Delta_{P} / h \sim 1 \mathrm{MHz}$ (here, $h$ is the Planck constant). We assert a compensating bias $\epsilon_{1}=2 \tilde{J}_{P}$ to this qubit. We dwell at this point for a time $\tau$, complete the anneal $s \rightarrow 1$ for the system plus probe, and then readout the state of the probe qubit. Figure 7 summarizes these waveforms during a typical QTS measurement.

We perform this measurement for a range of $\tau$, which allows us to measure an initial rate of tunneling $\Gamma$ from $\left|\psi_{0}^{L}, \downarrow\right\rangle$ to $|\psi, \uparrow\rangle$. We repeat this measurement of $\Gamma$ for a range of the probe qubit bias $\epsilon_{P}$. Peaks in $\Gamma$ correspond to resonances between the initially prepared state and the state $|n, \uparrow\rangle$, thus allowing us to map the eigenspectrum of the $N$-qubit system.

For the plots in the main paper, measurements of $\Gamma$ are normalized to $[0,1]$ by dividing $\Gamma$ by the maximum value across a vertical slice to give a visually interpretable result. Figure 8(b) shows a typical raw result in units of $\mu s^{-1}$.

We pose ferromagnetically coupled instances of the form

$$
\mathcal{H}_{P}=-\sum_{i} h_{i} \sigma_{i}^{z}+\sum_{i<j} J_{i j} \sigma_{i}^{z} \sigma_{j}^{z}
$$

with $J_{i j}<0$ for two- and eight-qubit subsections of the QA processor. Figure 8(a) shows typical measurements of $\Gamma$ for

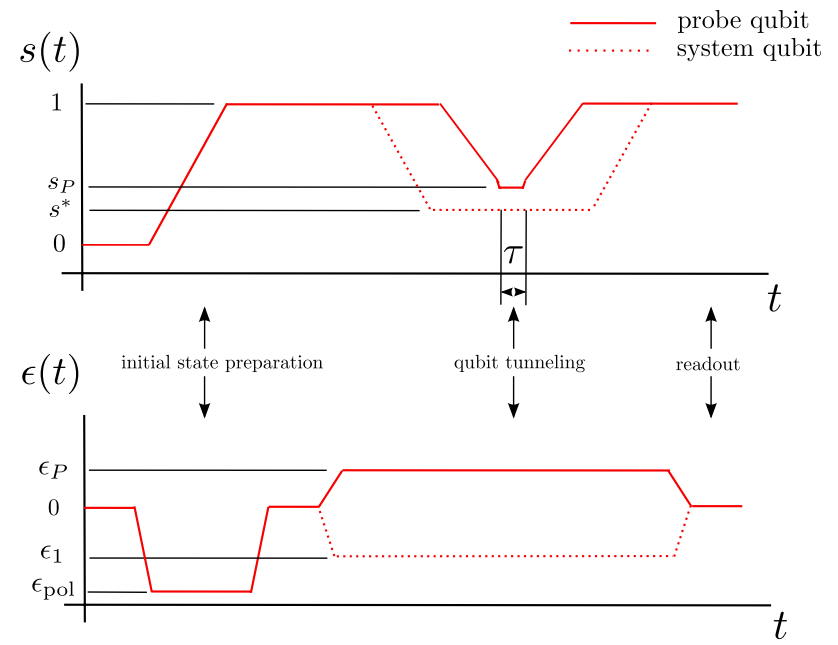

FIG. 7. Typical waveforms during QTS. We prepare the initial state by annealing probe and system qubits from $s=0$ to $s=1$ in the presence of a large polarization bias $\epsilon_{\text {pol }}$. We then bias the system qubit $q_{1}$ (to which the probe is attached) to a bias $\epsilon_{1}$ and the probe qubit to a bias $\epsilon_{P}$. With these biases asserted, we then adjust the system qubits' annealing parameter to an intermediate point $s^{*}$ and the probe qubit to a point $s_{P}$ and dwell for a time $\tau$. Finally, we complete the anneal $s \rightarrow 1$ and readout the state of the qubits. 


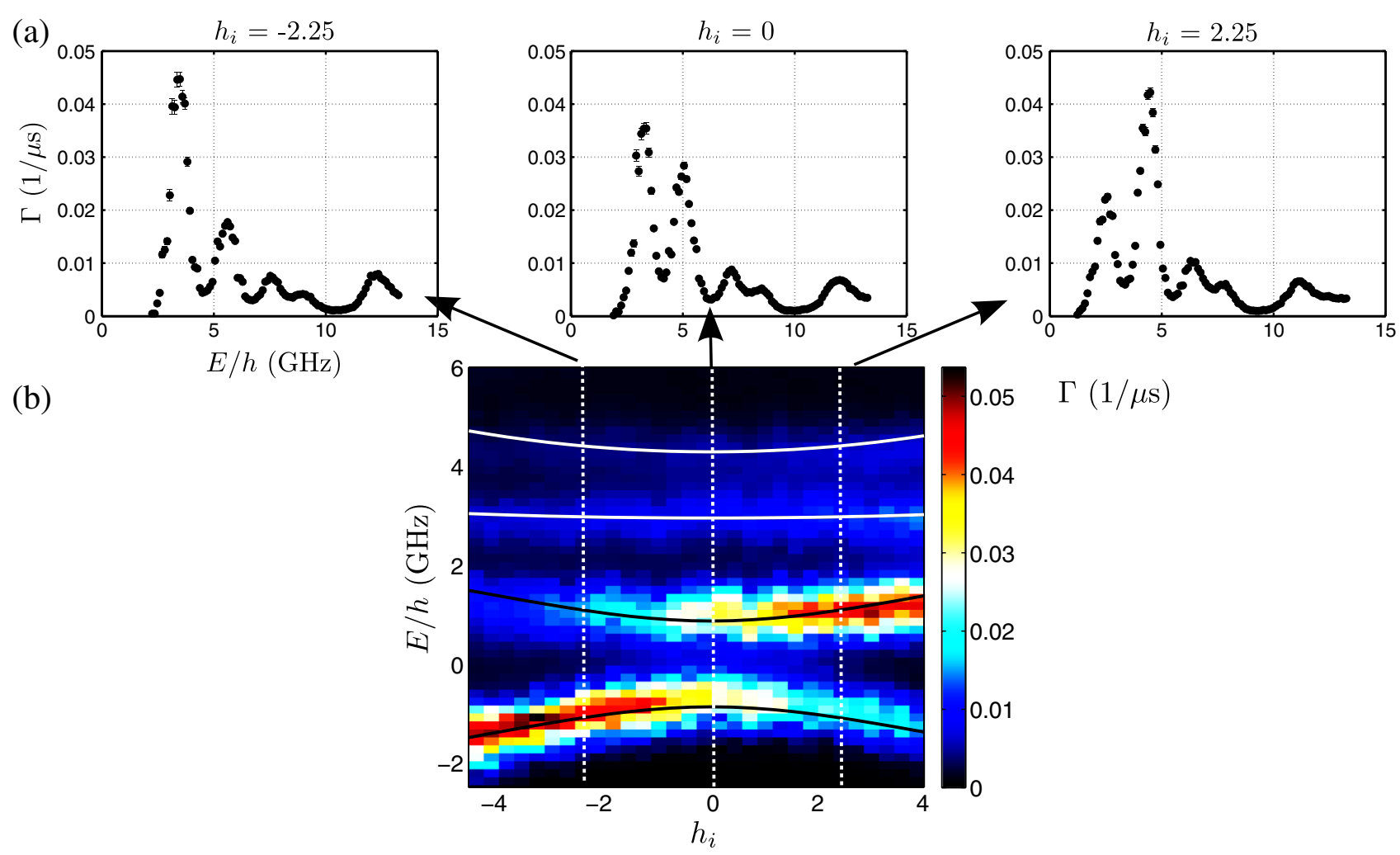

FIG. 8. Spectroscopy data for two FM-coupled qubits at $\tilde{J}_{P}<0$. (a) Measurements of tunneling rate $\Gamma$ for three values of $h_{1}=h_{2} \equiv h_{i}$. These data were taken at $s=0.339$. Peaks in $\Gamma$ reveal the energy eigenstates of the two-qubit system. (b) Multiple scans of $\Gamma$ for different values of $h_{i}$ assembled into a two-dimensional color plot. For better interpretability, we have subtracted off a baseline energy with respect to (a) such that the ground and first excited levels are symmetric about zero. Note the avoided crossing at $h_{i}=0$. The peak tunneling rate $\Gamma \sim\left|\Delta_{P}\left\langle\psi_{0}^{L} \mid n\right\rangle\right|^{2}$ [33]. The solid black and white curves plot the theoretical expectations for the energy eigenvalues using independent measurements shown in Fig. 6 and Hamiltonian (1).

a two-qubit subsection at several biases $h_{i}$ and at $s=0.339$ $\left(\tilde{J}_{P}<0\right)$. We assemble multiple measurements to produce the spectrum shown in Fig. 8(b).

\section{APPENDIX C: EQUILIBRIUM DISTRIBUTION OF SYSTEM}

In addition to the energy eigenspectrum, QTS also provides a means of measuring the equilibrium distribution of an $N$-qubit system with a probe qubit. Suppose we are in the limit $\left|\tilde{J}_{P}\right| \gg k_{B} T$, such that there is only one accessible state in the $|\downarrow\rangle_{P}$ manifold: $\left|\psi_{0}^{L}\right\rangle \otimes|\downarrow\rangle_{P}$. As described above, the other available states in the system are the composite eigenstates $|n\rangle \otimes|\uparrow\rangle_{P}$ in the $|\uparrow\rangle_{P}$ manifold, where $|n\rangle$ is an eigenstate of the $N$-qubit system without the probe qubit attached. Energy levels $E_{n}^{R}$ of the $|\uparrow\rangle_{P}$ manifold coincide with the energy levels $E_{n}$ of the system, $E_{n}^{R}=E_{n}$, even in the presence of coupling between the probe qubit and the system. We make the assumption that the population of an eigenstate depends only on its energy. Degenerate states have the same population.
Let $P^{L}$ represent the probability of finding the probe plus system in the state $\left|\psi_{0}^{L}\right\rangle \otimes|\downarrow\rangle_{P}$ and let $P_{n}^{R}$ represent the probability of finding the probe plus system in the state $|n\rangle \otimes|\uparrow\rangle_{P}$. At any point in the probe plus system evolution, we expect

$$
P^{L}+\sum_{i=1}^{2^{N}} P_{i}^{R}=1
$$

As described in the previous section, we can alter the energy of $\left|\psi_{0}^{L}\right\rangle \otimes|\downarrow\rangle_{P}$ with the probe bias $\epsilon_{P}$. Based on the spectroscopic measurements of the $N$-qubit eigenspectrum, we can choose an $\epsilon_{P}$ such that $\left|\psi_{0}^{L}\right\rangle \otimes|\downarrow\rangle_{P}$ and $|n\rangle \otimes|\uparrow\rangle_{P}$ are degenerate. Since the occupation of the state depends on its energy, we expect that, after long evolution times, these two degenerate states are occupied with equal probability, $P^{L}\left(\epsilon_{P}=E_{n}\right)=P_{n}^{R}$. Aligning the state $\left|\psi_{0}^{L}\right\rangle \otimes|\downarrow\rangle_{P}$ with all possible $2^{N}$ states $|n\rangle \otimes|\uparrow\rangle_{P}$, we obtain a set of relative probabilities $P_{n}^{R}$. These relative probabilities characterize the population distribution in the system since they are uniquely determined by the energy spectrum $E_{n}$. However, 
as follows from Eq. (C1), the set $P_{n}^{R}$ is not properly normalized. The probability distribution of the system itself is given by

$$
P_{n}\left(E_{n}\right)=\frac{P_{n}^{R}}{\sum_{i=1}^{2^{N}} P_{i}^{R}},
$$

where $\sum_{n=1}^{2^{N}} P_{n}=1$. At every eigenenergy, $\epsilon_{P}=E_{n}$, the denominator of Eq. (C2) can be found from Eq. (C1), so that the population distribution of the system $P_{n}$ has the form

$$
P_{n}=\frac{P_{n}^{R}}{1-P^{L}}=\frac{P^{L}\left(\epsilon_{P}=E_{n}\right)}{1-P^{L}\left(\epsilon_{P}=E_{n}\right)} .
$$

Thus, the probability $P_{n}$ to find the system of $N$ qubits in the state with energy $E_{n}$ can be estimated by measuring $P^{L}$ at $\epsilon_{P}=E_{n}$ and using Eq. (C3).

Measurements of $P^{L}$ proceed as they do for the spectroscopy measurements. The system plus probe is prepared in $\left|\psi_{0}^{L}, \downarrow\right\rangle$. We then adjust $\epsilon_{P}=E_{n}$ and an annealing parameter $s$ for the $N$-qubit system to some intermediate point, and also $s_{P}=0.612$ for the probe qubit, such that $\Delta_{P} / h \sim 1 \mathrm{MHz}$. We dwell at this point for a time $\tau \gg 1 / \Gamma$, complete the anneal $s \rightarrow 1$, and then readout the state of the probe qubit. We typically investigate a range of $\tau$ to ensure that we are in the long evolution time limit in which $P^{L}$ is independent of $\tau$. We use $P^{L}$ measured with $\tau=7041 \mu$ s to estimate $P_{1}$ and $P_{2}$. The Supplemental Material [34] contains typical data used for these estimates.

\section{APPENDIX D: ENTANGLEMENT MEASURES AND WITNESSES}

\section{Definition of entanglement}

A pure state $|\Psi\rangle$ of a system $S$ consisting of two parts $A$ and $B, S=A \cup B$, is entangled [16] with relation to this bipartition if the state $|\Psi\rangle$ cannot be represented as a product of states $\left|\Psi_{A}\right\rangle$ and $\left|\Psi_{B}\right\rangle$ describing the subsystems $A$ and $B:|\Psi\rangle \neq\left|\Psi_{A}\right\rangle \otimes\left|\Psi_{B}\right\rangle$. An open quantum system is characterized by a density matrix $\rho$. An open system $S$ is entangled [16] relative to the bipartition $S=A \cup B$ if its density matrix $\rho$ cannot be written as a convex sum of product states $\rho_{A}^{k} \otimes \rho_{B}^{k}, \rho \neq \sum_{k} w_{k} \rho_{A}^{k} \otimes \rho_{B}^{k}$. Here, $\left\{\rho_{A}^{k}\right\}$ and $\left\{\rho_{B}^{k}\right\}$ are sets of density matrices for the components $A$ and $B$, respectively; $w_{k} \geq 0, \sum_{k} w_{k}=1$. If there is no bipartition for which the system is entangled, the state is completely separable or unentangled. If the system is entangled for all possible bipartitions, the state is globally entangled.

\section{2. $\mathcal{W}_{\chi}$ : A susceptibility-based, ground-state entanglement witness}

For a bipartion of the system into two parts, $A$ and $B$, we define a witness $\mathcal{R}_{A B}$ as

$$
\mathcal{R}_{A B}=\frac{1}{4 N_{A B}}\left|\sum_{i \in A} \sum_{j \in B} \tilde{J}_{i j} \chi_{i j}\right|,
$$

where $\chi_{i j}$ is a cross susceptibility, $\tilde{J}_{i j}=\mathcal{E} J_{i j}$, and $N_{A B}$ is a number of nonzero couplings, $J_{i j} \neq 0$, between qubits from the subset $A$ and the subset $B$ (see Ref. [35]). We note that at low temperature, $T=12.5 \mathrm{mK}$, the measured susceptibility $\chi_{i j}(T)$ almost coincides with the ground-state susceptibility $\chi_{i j}(T=0)$ since contributions of excited states to $\chi_{i j}(T)$ are proportional to their populations, $P_{n} \ll 1$, for $n>1$. We analyze a deviation of the measured susceptibility from its ground-state value in the Supplemental Material [34]. To characterize global entanglement in the system of $N$ qubits, we introduce a witness $\mathcal{W}_{\chi}$,

$$
\mathcal{W}_{\chi}=\sqrt{\frac{\left(\prod \mathcal{R}_{A B}\right)^{1 / N_{p}}}{1+\left(\prod \mathcal{R}_{A B}\right)^{1 / N_{p}}}},
$$

which is given by a bounded geometrical mean of witnesses $\mathcal{R}_{A B}$ calculated for all possible partitions of the whole system into two subsystems. Here, $N_{p}$ is a number of such bipartitions, in particular, $N_{p}=127$ for the eightqubit ring.

\section{Thermal density matrix $\rho$}

Systems coupled to a thermal bath are in a mixed state when the energy gap between the ground and first excited states approaches $k_{B} T$. Most mixed-state entanglement measures and witnesses require knowledge of the density matrix $\rho$. The density matrix can be measured using quantum state tomography. However, this approach is limited to a small number of qubits [41]. As an alternate approach, we consider an $\mathrm{N}$-qubit system in a thermal (stationary) state [26]. The stationary system described by Hamiltonian (1) can be characterized by a density matrix that is diagonal in the energy basis, $\rho_{E}=\operatorname{diag}\left\{P_{1}\right.$, $\left.P_{2}, \ldots, P_{2^{N}}\right\}$. The off-diagonal elements of this matrix disappear on a very short decoherence time scale (within a few nanoseconds) in the quantum annealing processor analyzed in Ref. [6]. In thermal equilibrium, the occupation probability $P_{\mu}=e^{-E_{\mu} / k_{B} T} / Z$ is the Boltzmann distribution over the eigenstates $|\mu\rangle$ with energies $E_{\mu}$, such that $H|\mu\rangle=E_{\mu}|\mu\rangle, T$ is temperature, and $Z=\sum_{\mu} e^{-E_{\mu} / k_{B} T}$ is the partition function. The density matrix $\rho=$ $\sum \rho_{\bar{M}}|\bar{M}\rangle\langle\bar{N}|$ in the computation basis $|\bar{M}\rangle$, where $\rho_{\bar{M} \bar{N}}=\sum_{\mu} P_{\mu}\langle\bar{M} \mid \mu\rangle\langle\mu \mid \bar{N}\rangle$, is obtained from $\rho_{E}$ by a unitary transformation that depends on the parameters of the Hamiltonian $H$. The parameters of Hamiltonian (1), 
namely, the energy scale $\mathcal{E}$, the qubit biases $h_{i}$, tunneling amplitudes $\Delta$, coupling constants $J_{i j}$, as well as probabilities $P_{\mu}$, can be independently measured. This allows us to restore the density matrix $\rho$. We emphasize that the stationary-state entanglement, or thermal entanglement, is robust and does not decay with time [26].

\section{Concurrence $\mathcal{C}$}

For an open system described by a thermal density matrix $\rho$, concurrence $\mathcal{C}$ is defined as $[16,18]$

$$
\mathcal{C}=\max \left\{0, \sqrt{\lambda_{4}}-\sqrt{\lambda_{3}}-\sqrt{\lambda_{2}}-\sqrt{\lambda_{1}}\right\},
$$

where $\lambda_{4}>\lambda_{3}>\lambda_{2}>\lambda_{1}$ are the roots of the matrix

$$
\mathcal{R}=\rho\left(\sigma_{1}^{y} \otimes \sigma_{2}^{y}\right) \rho^{*}\left(\sigma_{1}^{y} \otimes \sigma_{2}^{y}\right),
$$

$\left\{\sigma_{i}^{x}, \sigma_{i}^{y}, \sigma_{i}^{z}\right\}$ are the Pauli matrices for the $i$ th qubit, and $\rho$ is the density matrix of the system in the computation basis.

An entanglement of formation $E_{f}$ is another measure of two-qubit entanglement. This measure is a monotonic function of concurrence $\mathcal{C}$ [16,18],

$$
E_{f}=\mathcal{F}\left(\frac{1+\sqrt{1-\mathcal{C}^{2}}}{2}\right)
$$

where $\mathcal{F}(x)=-x \log _{2}(x)-(1-x) \log _{2}(1-x) \quad$ is $\quad$ the entropy function.

\section{Negativity $\mathcal{N}$}

Negativity is a measure that provides a sufficient, but not necessary, condition for entanglement of an arbitrary number of qubits [36]. A nonzero value of negativity detects entanglement. To calculate negativity, we find all bipartitions of the system. For two qubits, there is only one bipartition. An eight-qubit system can be bipartitioned into $127(=8+28+56+35)$ possible combinations of two subsystems, $A$ and $B$. In the case when the state of the system is separable, its density matrix $\rho$ should retain all properties of the true density matrix after the partial transposition of $\rho$ with respect to the subsystem $A$ or to the subsystem $B[16,36]$. In particular, the partially transposed density matrix $\rho^{T_{A}}$ should not have negative eigenvalues. The negativity, $\mathcal{N}=(1 / 2) \sum_{i}\left(\left|\lambda_{i}\right|-\lambda_{i}\right)$, is proportional to the sum of all negative eigenvalues $\lambda_{i}$ of the matrix $\rho^{T_{A}}$, thus quantifying a degree of entanglement of the subsystem $A$ and the subsystem $B$. For the eight-qubit system, we analyze negativities for all 127 partitions, $\mathcal{N}_{1 / 7}, \mathcal{N}_{2 / 6}, \mathcal{N}_{3 / 5}, \mathcal{N}_{4 / 4}$, and calculate their geometrical average [42], or the global negativity, $\mathcal{N}(\rho)=$ $\left(\mathcal{N}_{1 / 7} \mathcal{N}_{2 / 6} \mathcal{N}_{3 / 5} \mathcal{N}_{4 / 4}\right)^{1 / 127}$. Here, the negativity $\mathcal{N}_{1 / 7}$ is equal to the product of 8 negativities for all possible partitions of the eight-qubit system into subsystems of one and seven qubits, and so on. Nonzero values of the global negativity mean that all possible subsystems of the whole eight-qubit system are globally entangled. Note that the negativity of the maximally entangled GHZ state, $\mathcal{N}_{\mathrm{GHZ}}$, is equal to $1 / 2$.

\section{Entanglement witness $\mathcal{W}_{A B}$}

Consider Hamiltonian (1) with measured parameters. This Hamiltonian describes a transverse Ising model having $N$ qubits. The ground state $\left|\psi_{1}\right\rangle$ of this model is entangled with respect to some bipartition $A-B$ of the $N$-qubit system. We can form an operator $\left|\psi_{1}\right\rangle\left\langle\psi_{1}||_{A}\right.$, where $T_{A}$ is a partial transposition operator with respect to the $A$ subsystem [16]. Let $|\phi\rangle$ be the eigenstate of $\left|\psi_{1}\right\rangle\left\langle\left.\psi_{1}\right|^{T_{A}}\right.$ with the most negative eigenvalue. We can form a new operator $\mathcal{W}_{A B}=|\phi\rangle\left\langle\left.\phi\right|^{T_{A}}\right.$. This operator can serve as an entanglement witness (it is trivially positive on all separable states).

Let $\rho(s)$ be the density matrix associated with the state of the system at the annealing point $s$. If we have experimental measurements of the occupation fraction of the ground state and first excited state, $P_{1}(s) \pm \delta P_{1}$ and $P_{2}(s) \pm \delta P_{2}$, respectively, we can place a set of linear constraints on $\rho(s)$ :

$$
\begin{aligned}
& \operatorname{Tr}\left[\rho(s)\left|\psi_{1}\right\rangle\left\langle\psi_{1}\right|\right] \geq P_{1}(s)-\delta P_{1}, \\
& \operatorname{Tr}\left[\rho(s)\left|\psi_{1}\right\rangle\left\langle\psi_{1}\right|\right] \leq P_{1}(s)+\delta P_{1}, \\
& \operatorname{Tr}\left[\rho(s)\left|\psi_{2}\right\rangle\left\langle\psi_{2}\right|\right] \geq P_{2}(s)-\delta P_{2}, \\
& \operatorname{Tr}\left[\rho(s)\left|\psi_{2}\right\rangle\left\langle\psi_{2}\right|\right] \leq P_{2}(s)+\delta P_{2} .
\end{aligned}
$$

We now search over all possible $\rho(s)$ that satisfy the linear constraints provided by the experimental data. The goal is to maximize the witness $\operatorname{Tr}\left[\mathcal{W}_{A B} \rho(s)\right]$ in order to establish an upper limit for this quantity. Maximizing this quantity can be cast as a semidefinite program [37], a class of convex optimization problems for which efficient algorithms exist. When this upper limit is less than zero, entanglement is certified for the bipartition $A-B$.

We test the robustness of this result with uncertainties in the parameters of the Hamiltonian. To do this, we repeat the analysis at several points during the QA algorithm when adding random perturbations on the measured Hamiltonian that correspond to the uncertainty on these measured quantities. We sample $10^{4}$ perturbed Hamiltonians and, for every perturbation, the optimization results in $\operatorname{Tr}\left[\mathcal{W}_{A B} \rho(s)\right]<0$.

[1] M. Mariantoni, H. Wang, T. Yamamoto, M. Neeley, R. C. Bialczak, Y. Chen, M. Lenander, E. Lucero, A. D. O'Connell, D. Sank, M. Weides, J. Wenner, Y. Yin, J. Zhao, A. N. Korotkov, A. N. Cleland, and J. M. Martinis, Implementing the Quantum von Neumann Architecture with Superconducting Circuits, Science 334, 61 (2011). 
[2] E. Lucero, R. Barends, Y. Chen, J. Kelly, M. Mariantoni, A. Megrant, P. O’Malley, D. Sank, A. Vainsencher, J. Wenner, T. White, Y. Yin, A. N. Cleland, and J. M. Martinis, Computing Prime Factors with a Josephson Phase Qubit Quantum Processor, Nat. Phys. 8, 719 (2012).

[3] M. D. Reed, L. DiCarlo, S. E. Nigg, L. Sun, L. Frunzio, S. M. Girvin, and R. J. Schoelkopf, Realization of ThreeQubit Quantum Error Correction with Superconducting Circuits, Nature (London) 482, 382 (2012).

[4] A. G. Fowler, M. Mariantoni, J. M. Martinis, and A. N. Cleland, Surface Codes: Towards Practical Large-Scale Quantum Computation, Phys. Rev. A 86, 032324 (2012).

[5] T. S. Metodi, D. D. Thaker, and A. W. Cross, Proceedings of the 38th Annual IEEE/ACM International Symposium on Microarchitecture (MICRO-38), 2005 (IEEE, New York, 2005).

[6] M. W. Johnson et al., Quantum Annealing with Manufactured Spins, Nature (London) 473, 194 (2011).

[7] N.G. Dickson et al., Thermally Assisted Quantum Annealing of a 16-Qubit Problem, Nat. Commun. 4, 1903 (2013).

[8] R. Harris et al., Experimental Investigation of an EightQubit Unit Cell in a Superconducting Optimization Processor, Phys. Rev. B 82, 024511 (2010).

[9] R. Blatt and D. Wineland, Entangled States of Trapped Atomic Ions, Nature (London) 453, 1008 (2008).

[10] T. Monz, P. Schindler, J. T. Barreiro, M. Chwalla, D. Nigg, W. A. Coish, M. Harlander, W. Hänsel, M. Hennrich, and R. Blatt, 14-Qubit Entanglement: Creation and Coherence, Phys. Rev. Lett. 106, 130506 (2011).

[11] M. Ansmann, H. Wang, R. C. Bialczak, M. Hofheinz, E. Lucero, M. Neeley, A. D. O'Connell, D. Sank, M. Weides, J. Wenner, A. N. Cleland, and J.M. Martinis, Violation of Bell's Inequality in Josephson Phase Qubits, Nature (London) 461, 504 (2009).

[12] M. Neeley, R. C. Bialczak, M. Lenander, E. Lucero, M. Mariantoni, A. D. O'Connell, D. Sank, H. Wang, M. Weides, J. Wenner, Y. Yin, T. Yamamoto, A. N. Cleland, and J. M. Martinis, Generation of Three-Qubit Entangled States Using Superconducting Phase Qubits, Nature (London) 467, 570 (2010).

[13] L. DiCarlo, M. D. Reed, L. Sun, B. R. Johnson, J. M. Chow, J. M. Gambetta, L. Frunzio, S. M. Girvin, M. H. Devoret, and R. J. Schoelkopf, Preparation and Measurement of Three-Qubit Entanglement in a Superconducting Circuit, Nature (London) 467, 574 (2010).

[14] A. J. Berkley, H. Xu, R. C. Ramos, M. A. Gubrud, F. W. Strauch, P. R. Johnson, J. R. Anderson, A. J. Dragt, C. J. Lobb, and F. C. Wellstood, Entangled Macroscopic Quantum States in Two Superconducting Qubits, Science 300, 1548 (2003).

[15] G. Vidal, Efficient Classical Simulation of Slightly Entangled Quantum Computations, Phys. Rev. Lett. 91, 147902 (2003).

[16] O. Gühne and G. Tóth, Entanglement Detection, Phys. Rep. 474, 1 (2009).

[17] D. Greenberger, M. Horne, A. Shimony, and A. Zeilinger, Bells Theorem without Inequalities, Am. J. Phys. 58, 1131 (1990).
[18] W. K. Wootters, Entanglement of Formation of an Arbitrary State of Two Qubits, Phys. Rev. Lett. 80, 2245 (1998).

[19] A. B. Finnila, M. A. Gomez, C. Sebenik, C. Stenson, and J. D. Doll, Quantum Annealing: A New Method for Minimizing Multidimensional Functions, Chem. Phys. Lett. 219, 343 (1994).

[20] T. Kadowaki and H. Nishimori, Quantum Annealing in the Transverse Ising Model, Phys. Rev. E 58, 5355 (1998).

[21] E. Farhi, J. Goldstone, S. Gutmann, J. Lapan, A. Lundgren, and D. Preda, A Quantum Adiabatic Evolution Algorithm Applied to Random Instances of an NP-Complete Problem, Science 292, 472 (2001).

[22] G. E. Santoro, R. Martonak, E. Tosatti, and R. Car, Theory of Quantum Annealing of an Ising Spin Glass, Science 295, 2427 (2002).

[23] A. Perdomo-Ortiz, N. Dickson, M. Drew-Brook, G. Rose, and A. Aspuru-Guzik, Finding Low-Energy Conformations of Lattice Protein Models by Quantum Annealing, Sci. Rep. 2, 571 (2012).

[24] S. Boixo, T. Albash, F. M. Spedalieri, N. Chancellor, and D. A. Lidar, Experimental Signature of Programmable Quantum Annealing, Nat. Commun. 4, 2067 (2013).

[25] R. Harris, T. Lanting, A. J. Berkley, J. Johansson, M. W. Johnson, P. Bunyk, E. Ladizinsky, N. Ladizinsky, T. Oh, and S. Han, A Compound Josephson Junction Coupler for Flux Qubits with Minimal Cross Talk, Phys. Rev. B 80, 052506 (2009).

[26] L. Amico, R. Fazio, A. Osterloch, and V. Vedral, Entanglement in Many-Body Systems, Rev. Mod. Phys. 80, 517 (2008).

[27] X. Wang, Thermal and Ground-State Entanglement in Heisenberg XX Qubit Rings, Phys. Rev. A 66, 034302 (2002).

[28] S. Ghosh, T. F. Rosenbaum, G. Aeppli, and S. N. Coppersmith, Entangled Quantum State of Magnetic Dipoles, Nature (London) 425, 48 (2003).

[29] T. Vértesi and E. Bene, Thermal Entanglement in the Nanotubular System $\mathrm{Na}_{2} \mathrm{~V}_{3} \mathrm{O}_{7}$, Phys. Rev. B 73, 134404 (2006).

[30] C. Brukner, V. Vedral, and A. Zeilinger, Crucial Role of Quantum Entanglement in Bulk Properties of Solids, Phys. Rev. A 73, 012110 (2006).

[31] T. G. Rappoport, L. Ghivelder, J. C. Fernandes, R. B. Guimaraes, and M. A. Continentino, Experimental Observation of Quantum Entanglement in Low-Dimensional Systems, Phys. Rev. B 75, 054422 (2007).

[32] N. B. Christensen, H. M. Ronnow, D. F. McMorrow, A. Harrison, T. G. Perring, M. Enderle, R. Coldea, L. P. Regnault, and G. Aeppli, Quantum Dynamics and Entanglement of Spins on a Square Lattice, Proc. Natl. Acad. Sci. U.S.A. 104, 15264 (2007).

[33] A. J. Berkley, A. J. Przybysz, T. Lanting, R. Harris, N. Dickson, F. Altomare, M. H. Amin, P. Bunyk, C. Enderud, E. Hoskinson, M. W. Johnson, E. Ladizinsky, R. Neufeld, C. Rich, A. Y. Smirnov, E. Tolkacheva, S. Uchaikin, and A. B. Wilson, Tunneling Spectroscopy Using a Probe Qubit, Phys. Rev. B 87, 020502 (2013).

[34] See Supplemental Material at http://link.aps.org/ supplemental/10.1103/PhysRevX.4.021041 includes 
additional information on susceptibility and spectroscopy measurements, additional detail on data analysis, and a discussion of the susceptiblity-based witness at finite temperature.

[35] A. Yu. Smirnov and M. H. Amin, Ground-State Entanglement in Coupled Qubits, Phys. Rev. A 88, 022329 (2013).

[36] G. Vidal and R.F. Werner, A Computable Measure of Entanglement, Phys. Rev. A 65, 032314 (2002).

[37] F. M. Spedalieri, Detecting Entanglement with Partial State Information, Phys. Rev. A 86, 062311 (2012).

[38] R. Harris, J. Johansson, A. J. Berkley, M. W. Johnson, T. Lanting, S. Han, P. Bunyk, E. Ladizinsky, T. Oh, I. Perminov, E. Tolkacheva, S. Uchaikin, E. M. Chapple, C. Enderud, C. Rich, M. Thom, J. Wang, B. Wilson, and G. Rose, Experimental Demonstration of a Robust and Scalable Flux Qubit, Phys. Rev. B 81, 134510 (2010).
[39] J. Johansson, M. H. S. Amin, A. J. Berkley, P. Bunyk, V. Choi, R. Harris, M. W. Johnson, T. M. Lanting, S. Lloyd, and G. Rose, Landau-Zener Transitions in a Superconducting Flux Qubit, Phys. Rev. B 80, 012507 (2009).

[40] R. Harris, M. W. Johnson, S. Han, A. J. Berkley, J. Johansson, P. Bunyk, E. Ladizinsky, S. Govorkov, M. C. Thom, S. Uchaikin, B. Bumble, A. Fung, A. Kaul, A. Kleinsasser, M. H. S. Amin, and D. V. Averin, Probing Noise in Flux Qubits via Macroscopic Resonant Tunneling, Phys. Rev. Lett. 101, 117003 (2008).

[41] M. P. da Silva, O. Landon-Cardinal, and D. Poulin, Practical Characterization of Quantum Devices without Tomography, Phys. Rev. Lett. 107, 210404 (2011).

[42] P. J. Love, A. M. van den Brink, A. Yu. Smirnov, M. H. S. Amin, M. Grajcar, E. Il'ichev, A. Izmalkov, and A. M. Zagoskin, A Characterization of Global Entanglement, Quantum Inf. Process. 6, 187 (2007). 